\title{
AVALIAÇÃO DA IMAGEM CORPORAL EM PROFESSORES DE EDUCAÇÃO FÍSICA ATUANTES NO FITNESS NA CIDADE DO RIO DE JANEIRO'
}

\author{
MS. LÍGIA MARTINS VILHENA \\ Mestre em Educação Física pela Universidade Gama Filho (UGF) e colaboradora do \\ Laboratório de Educação em Ambiente e Saúde (LEAS), Fundação e Instituto Oswaldo Cruz \\ (FIOCRUZ) (Rio de Janeiro - Rio de Janeiro - Brasil) \\ E-mail: ligiavilhena@ig.com.br \\ DR. TONY MEIRELES SANTOS \\ Doutor em Educação Física e Docente do Programa de Pós-Graduação em \\ Educação Física (PPGEF-UGF), Universidade Gama Filho \\ (Rio de Janeiro - Rio de Janeiro - Brasil) \\ E-mail: tonyms@prohealth.com.br \\ DR. ALEXANDRE PALMA \\ Doutor em Saúde Pública pela Fundação Oswaldo Cruz (FIOCRUZ) e professor adjunto da \\ Universidade Federal do Rio de Janeiro (Rio de Janeiro - Rio de Janeiro - Brasil) \\ E-mail: palma_alexandre@yahoo.com.br

\section{DRA. LUDMILA MURÃO} \\ Doutora em Educação Física pela Universidade Gama Filho (UGF) e professora adjunta da \\ Universidade Federal de Juiz de Fora (Juiz de Fora - Minas Gerais - Brasil) \\ E-mail: ludmila.mourao@terra.com.br
}

I. O presente trabalho contou com apoio financeiro do Conselho Nacional de Desenvolvimento Científico e Tecnológico (CNPq). 


\section{RESUMO}

Identificar a prevalência de distorções de imagem corporal (IC) entre os sexos, em professores atuantes no mercado do Fitness da cidade do Rio de Janeiro. Para avaliação da IC foi utilizado o Body Shape Questionnaire (BSQ) em 255 professores (I 79 homens). Observamos que 7,3\% dos professores e 32,8\% das professoras apresentaram leve ou moderada distorção. As mulheres $(71,2 \pm 22,4)$ apresentaram escores de BSQ estatisticamente superiores $(p<0,001)$ aos homens $(53,2 \pm 17,0)$. Observou-se ainda uma crescente preocupação e cuidado com a construção de uma forma ideal de corpo entre os docentes, uma vez que níveis elevados de desempenho físico e estético significam garantia de domínio técnico e psicológico junto aos alunos, impactando em sua moral e sucesso.

PALAVRAS-CHAVE: Dieta; substâncias químicas; docente; estética.

\section{INTRODUÇÃO}

$\mathrm{Na}$ atualidade, o corpo parece ter grande potencial para o consumo, para a experimentação biotecnológica e para os investimentos econômicos (LEAL et al., 20 I0). Mídia, empresas e órgãos governamentais encorajam as pessoas a se exercitarem em nome da saúde e da qualidade de vida. No entanto, pesquisas recentes ainda buscam identificar os principais motivos de adesão (SILVA LOPES; CHIAPETA, 20 I 0) e desistência (LIZ et al., 20 I0) de brasileiros praticantes de exercícios físicos em academias de ginástica. Dentre as razões mencionadas para a adesão, destacaram-se a busca pela saúde, estética, socialização, melhoria da condição física e bem-estar. A desistência foi atribuída à falta de tempo, preguiça, distância que o praticante deverá percorrer do seu trabalho ou da sua casa até a academia e também ao alto custo financeiro das atividades (LIZ et al. 20 I0).

Diante da dificuldade de identificação e administração destes fatores para a promoção da atividade física e da saúde, cada vez mais busca-se informações específicas sobre esse mercado em franca expansão. $\bigcirc$ Instituto Fitness Brasil ${ }^{2}$ revelou, por intermédio de dados coletados pelo Conselho Federal de Educação Física (CONFEF), que em 2008 o Brasil tinha um total de 12.682 academias, 3,9

2. O Instituto Fitness Brasil é uma $O N G$ que tem como missão oferecer saúde e bem-estar à sociedade através do fornecimento de conteúdo, do desenvolvimento e atualização de profissionais, da atuação em políticas públicas e do aval de qualidade para produtos e serviços ligados ao bem-estar. Acessado em 17//0/2010 no endereço eletrônico: http://www.pactosolucoes. $\mathrm{com} \cdot \mathrm{br} / \mathrm{blog} /$ ?tag = ihrsa-fitness-brasil 
milhões de clientes e US $\$$ I,2 bilhão em receita. Além das atividades físicas, a cultura fitness oferece uma diversidade de bens e serviços destinados ao aperfeiçoamento da aptidão física, da sedução e do prazer. Contudo, este contexto é revelado não apenas como a aquisição de capacidades orgânicas, como também com propósitos comerciais manifestados por um estilo de ser e viver entre as pessoas daquele grupo (GOELLNER, 2008). A autora ainda alega que "são os objetivos estéticos que têm empurrado as mulheres para a exercitação de seus corpos e a modelagem de suas aparências" (GOELLNER, 2008, p. 250).

Estudos sugerem que as mulheres parecem demonstrar maiores preocupações com o corpo, quando comparadas aos homens (SECCHI; CAMARGO; BERTOLDO, 2009; HAY, 2002). Thompson et al. (1995) apontam que, na opinião da mulher contemporânea, o baixo peso corporal representa o ideal de beleza feminina, além de ser um fator determinante de aceitação e êxito pessoal. Já entre os homens, a expectativa é de corpos musculosos e com baixo percentual de gordura. Segundo Goldenberg e Ramos (2002), o corpo é um valor que distingue os indivíduos, especialmente em cidades litorâneas como o Rio de Janeiro. No entanto, a atual valorização do corpo magro não corresponde ao padrão corporal das mulheres brasileiras, nem mesmo das cariocas. Nesse sentido, a pesquisa realizada em 2008 pelo Ministério da Saúde nas capitais brasileiras, estabeleceu a frequência e distribuição sociodemográfica de fatores de risco e proteção para doenças crônicas, revelando que: 42,3\% das mulheres da cidade do Rio de Janeiro estão acima do peso, com índice de massa corporal (IMC) igual ou superior a 25 $\mathrm{kg} \cdot \mathrm{m}^{-2}$. A investigação ainda apontou que a maior frequência de excesso de peso foi observada em homens na cidade de Rio Branco (56,3\%), e nas mulheres da cidade de Cuiabá (44,8\%).

Grande parte dos estudos sobre a IC tem utilizado avaliações de percepções corporais em populações clínicas com tendências a distúrbios psiquiátricos, em especial, os transtornos alimentares como a bulimia e a anorexia nervosas. As síndromes ocorrem, em sua maioria, entre mulheres jovens, ocidentais e, sobretudo, com históricos de transtornos psiquiátricos associados à adoção de dieta, vulnerabilidade pessoal e ambiental (HAY, 2002). São recentes as investigações sobre a prevalência de distúrbios de IC em populações que podem estar suscetíveis ou vulneráveis a rígidos padrões corporais, como por exemplo, atletas (OLIVEIRA et al., 2003) e estudantes universitários (MOREIRA et al., 2005).

Segundo a classificação do Manual de Diagnóstico e Estatística da Associação Psiquiátrica Americana, outro tipo de distúrbio conhecido por dismorfia muscular, presente quase exclusivamente em homens, manifesta-se quando o indivíduo hipertrofiado procura aumentar a massa muscular através de exercícios excessivos, do 
consumo de substancias ergogênicas e pela adoção de dietas hiperprotéicas (SARDINHA; OLIVEIRA; ARAÚJO, 2008). Ainda assim, estes indivíduos apresentam-se insatisfeitos com sua aparência.

O estudo de Palma e Assis (2005) constatou que 38,7\% dos professores de educação física atuantes no fitness já fizeram uso de aceleradores metabólicos e 25,6\% de esteróides anabólico-androgênicos (EAA), sendo 30,5\% do sexo masculino e 12,2\% do sexo feminino. Os discursos dos docentes indicaram que o uso foi motivado pela preocupação com o aumento da resistência ao esforço prolongado, e também pela necessidade de conquistar o modelo de corpo hipertrofiado, valorizado no ambiente do fitness, principalmente no campo de atuação profissional como o da musculação. Diante de tais requisitos, é possível que a apresentação corporal dos professores(as) de educação física atuantes no fitness inspire confiança e credibilidade no exercício de sua função, uma vez que suas atuações têm a prerrogativa de modelagem dos corpos. Presumimos, então, que a auto-exigência em relação à estética corporal entre estes professores possa contribuir para o uso de substâncias ergogênicas, assim como a adoção de dietas contínuas e, consequentemente, para o desenvolvimento de distúrbios de IC (THOMPSON et al., 1995).

Neste sentido, os objetivos do presente estudo foram: a) identificar a prevalência de distorções de IC entre professores de educação física atuantes em academias de ginástica, acompanhando as diferenças entre os sexos; b) investigar a associação entre os escores do BSQ e IMC e c) averiguar a probabilidade do uso de anabolizantes, suplementos alimentares, produtos emagrecedores, assim como se a adoção de dietas influenciarem no desenvolvimento de distorção da IC entre os professores (as) atuantes no ambiente do fitness.

\section{MATERIAIS E MÉTODOS}

Os professores que se dispuseram a participar da pesquisa seguiram os seguintes critérios de inclusão: graduados em educação física de ambos os sexos registrados no Conselho Regional de Educação Física; idade acima de 24 anos; atuantes exclusivamente em academias de ginástica, em programas específicos de treinamento da estética corporal e/ou do condicionamento físico. Dessa forma, participaram do estudo 255 professores, sendo 179 (70,2\%) do sexo masculino e $76(29,8 \%)$ do feminino.

O número de professores foi calculado considerando a população estimada de 594 profissionais atuantes em 64 academias de ginástica na cidade do Rio de Janeiro. Para um erro amostral estipulado em cinco pontos percentuais, com nível de confiança de $95 \%$ e prevalência presumida de 50\%, foi estimado o tamanho 
amostral em 234 professores. Foram recrutados $\approx 275$ professores, superando em 15\% a amostra originalmente estimada. Para que fosse possível estimar o quantitativo de academias ativas na cidade do Rio de Janeiro, selecionamos as registradas na lista telefônica, através de consulta à internet (www.telelista.net). Foi identificado um total de 639 academias de ginástica de grande, médio e pequeno porte, sendo que: 64 ( $10 \%)$ delas foram sorteadas através da tabela de números aleatórios para participar do estudo. Em seguida, e através de contato telefônico, procurou-se investigar o número total de professores atuantes nas academias sorteadas. Pela opção de 24 academias (25\%) em não participar do estudo, dois novos sorteios foram realizados, resultando no convite a 96 academias para que alcançássemos nossa meta original (64).

Para o levantamento de dados, foi utilizado um questionário anônimo com perguntas sobre dados pessoais, questões sobre o consumo de esteróides anabólico-androgênicos, suplementos alimentares, a adoção e classificação de dietas. $\bigcirc$ peso e a estatura foram auto-reportados. Este procedimento mostrou alta fidedignidade no estudo de Maranhão Neto, Polito e Lira, (2005). Assim, o IMC foi estimado com base nesses valores. A parte que se referiu ao consumo de EAA e outras substâncias foi elaborada tendo como referência o questionário utilizado no estudo de Palma e Assis (2005).

Para detectar distúrbios relacionados à IC, foi utilizado o Body Shape Questionnaire (-BSQ), validado para a língua portuguesa no estudo de Cordás e Neves ( 1999), assim como para uma população de universitários brasileiros por Di Pietro (200 I). A consistência interna da escala brasileira medida através do alfa de Cronbach, foi de 0,97 , indicando que as questões convergem para o mesmo construto. A análise fatorial da escala resultou em uma solução de quatro dimensões da variabilidade total dos dados. Assim, a versão original do BSQ possui 34 itens designados para mensurar a satisfação e as preocupações com a forma do corpo, organizados numa escala tipo Likert de seis pontos, que varia de nunca a sempre.

somatório dos resultados indica a presença ou não de distorções. Considera-se que, quando a soma dos pontos é inferior a 80, há ausência de distorções de IC. Entre 8 I e I I 0 pontos, o sujeito apresenta leve distorção. Valores situados entre ||| e 140 classificam-no como de moderada distorção, e acima de 140 pontos, o sujeito é considerado com grave distorção de $\mathrm{IC}$.

Os questionários foram entregues, dentro de um envelope lacrado, nas academias sorteadas, mediante autorização do coordenador responsável. Mesmo sendo o instrumento auto-aplicável, a pesquisadora, sempre que possível, permaneceu no ambiente. Os questionários eram, então, devolvidos dentro do mesmo envelope, garantindo o anonimato dos informantes. 
Foi realizada a estatística descritiva através de distribuição de frequência em valores absolutos e relativos, média e desvio padrão. A normalidade dos dados foi verificada pelo teste de Shapiro Wilk. Para comparar os resultados de BSQ entre os sexos, foi utilizado o teste t para amostras independentes. A análise de correlação entre o BSQ e IMC foi verificada pelo coeficiente de correlação de Pearson. Complementarmente, foram reportadas as estimativas do intervalo de confiança utilizando a transformação $z$ de Fisher's, com precisão de 95\%, e conduzidas análises de razão de chance para desfechos específicos relacionados ao tema investigado. As análises foram realizadas no programa GraphPad Prism 5 (GraphPad software Inc., California, USA), com nível de significância em $p £ 0,05$.

\section{RESULTADOS}

Verificou-se que I 66 (92,7\%) dos professores e 5 I (67,2\%) das professoras não apresentaram distorção de IC; contudo, 13 (7,3\%) da amostra masculina e 25 (32,8\%) da amostra feminina apresentaram de leve a moderada distorção de IC. Foram observados, ainda, resultados estatisticamente significativos ( $p<0,00 \mathrm{I}$ ) nos escores de BSQ para as mulheres: 71,2 \pm 22,4, quando comparada aos homens: 53,2 \pm 17,0 (Figura I).

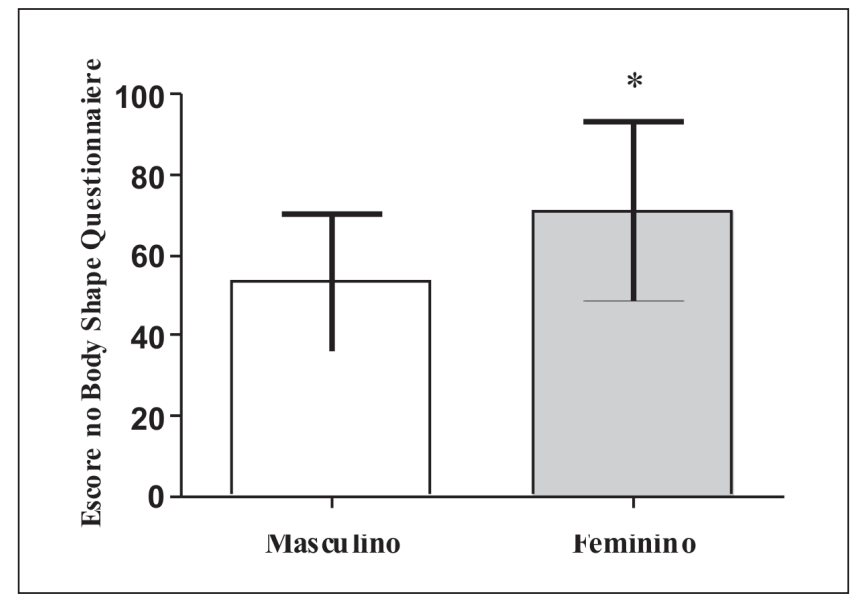

Figura I: Escores do Body Shape Questionnaire (BSQ) entre os sexos. * - diferença significativa para p < 0,05.

Os três painéis dispostos na Figura 2 representam as correlações entre IMC e os escores do BSQ de homens, mulheres e todos os sujeitos investigados. Em que pese a baixa correlação entre o IMC e a IC, observou-se associação 
significava somente para os homens ( $r=0,343$; IC95\% 0, I09-0,392; $p=$ $0,000)$, enquanto para as mulheres $(r=0,147,1 C 95 \%-0,041-0,406 ; p=$ $0,205)$ e para todo o grupo $(r=0,057, I C 95 \% 0,1$ I 8 - 0, I 34; $p=0,363)$ não foram observados resultados significativos.
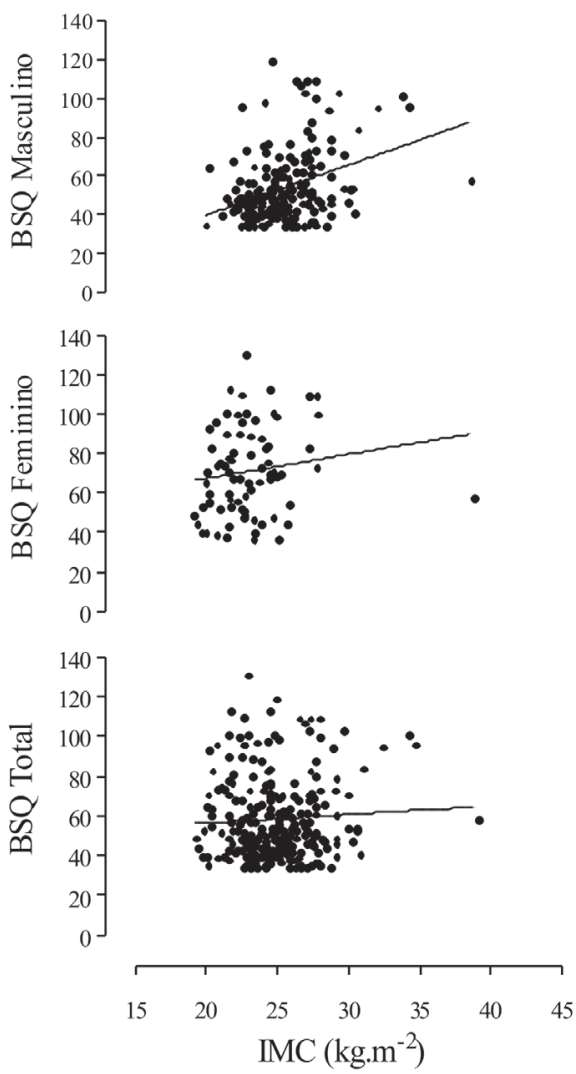

Figura 2: Associação entre o Body Shape Questionnaire (BSQ) e índice de massa corporal (IMC) para homens, mulheres e o total de sujeitos investigados.

A Tabela I permite identificar a razão de chance da adoção de práticas específicas com o propósito de modelar o corpo, e influenciar o desenvolvimento da distorção de IC. Os dados revelaram que a adoção da dieta hipocalórica influenciou significativamente o desenvolvimento da distorção de IC entre os professores e as professoras: $(\mathrm{OR}=4,17, \mathrm{p}<0,00 \mathrm{I})$. Foi observado, ainda, que não houve asso- 
ciação entre as condutas com o propósito de modelagem corporal e a distorção de IC entre os 194 (76, I\%) usuários de suplementos alimentares; os 86 (33,9\%) professores (as) que alegaram fazer uso de produtos emagrecedores; assim como para os 55 (21,6\%) usuários de EAA e, por fim, os 39 (15,3\%) professores que adotaram a dieta hipercalórica.

Tabela I - Associações entre as condutas adotadas com o propósito de modelar o corpo e a distorção da IC

\begin{tabular}{|c|c|c|c|c|c|c|}
\hline \multirow{3}{*}{ Condutas } & \multicolumn{4}{|c|}{ Distorção da Imagem Corporal } & \multirow{3}{*}{ OR } & \multirow{3}{*}{$\mathrm{p}$} \\
\hline & \multicolumn{2}{|c|}{ Sem } & \multicolumn{2}{|c|}{ Com } & & \\
\hline & $\mathrm{n}$ & $\%$ & $\mathrm{n}$ & $\%$ & & \\
\hline \multicolumn{7}{|c|}{ Produtos para emagrecer } \\
\hline Não fez uso & 148 & 88,1 & 20 & 11,9 & 1,00 & \multirow{2}{*}{0,056} \\
\hline Fez uso & 68 & 79,1 & 18 & 20,9 & 1,96 & \\
\hline \multicolumn{7}{|c|}{ Suplementos alimentares } \\
\hline Não fez uso & 50 & 82,0 & 11 & 18,0 & ।, 00 & \multirow{2}{*}{0,431} \\
\hline Fez uso & 167 & 86,1 & 27 & 13,9 & 0,73 & \\
\hline \multicolumn{7}{|c|}{ Esteróides anabólico-androgênicos } \\
\hline Não fez uso & 168 & 84,0 & 32 & 16,0 & ।, 00 & \multirow{2}{*}{0,348} \\
\hline Fez uso & 49 & 89,1 & 6 & 10,9 & 0,64 & \\
\hline \multicolumn{7}{|l|}{ Dieta hipocalórica } \\
\hline Não fez uso & 175 & 90,2 & 19 & 9,8 & 1,00 & \multirow[b]{2}{*}{$<0,00$} \\
\hline Fez uso & 42 & 68,9 & 19 & 31,1 & 4,17 & \\
\hline \multicolumn{7}{|l|}{ Dieta hipercalórica } \\
\hline Não fez uso & 183 & 84,7 & 33 & 15,3 & 1,00 & \multirow{2}{*}{0,692} \\
\hline Fez uso & 34 & 87,2 & 5 & 12,8 & 0,82 & \\
\hline
\end{tabular}

OR - razão de chance; p - nível de significância

\section{DISCUSSÃO DOS RESULTADOS}

Pesquisas sobre a insatisfação corporal feminina (SARDINHA; OLIVEIRA; ARAÚJO, 2008) sugerem que o conflito entre o corpo atual (real) e o corpo desejado parece estimular a adoção de dietas, e que, quando mantidas por períodos prolongados, pode culminar em distúrbios psiquiátricos, como os transtornos alimentares (OLIVA; FAGUNDES, 200 I; HAY, 2002). Tal descontentamento com o corpo foi verificado entre $64,4 \%$ de uma amostra de universitárias brasileiras, 
quando relataram que gostariam de estar com a figura corporal atual menor e, mesmo as estudantes com padrão de corpo adequado, escolheram figuras saudáveis e ideais menores (ALVARENGA et al., 20l0). Nessa direção, entre um grupo de atletas brasileiras de diferentes modalidades, o valor médio do BSQ foi de 77,8 \pm 28,7 (OLIVEIRA et al., 2003). Este achado encontra-se em discordância com a literatura, ao sugerir que indivíduos ativos apresentam IC mais positiva, independente da idade e do sexo (HAUSENBLAS; FALLON, 2006). A ideia acerca da reivindicação pela magreza, entre frequentadores de academias de ginástica, também foi reforçada quando as mulheres e indivíduos com maior percentual de gordura apresentaram maior insatisfação corporal (FERMINO; PEZZINI; REIS, 20I0).

No período em que foi realizada esta investigação, os resultados apontaram maior insatisfação e/ou preocupação em relação ao excesso de peso corporal também entre as professoras. Os investimentos com o propósito de alteração corporal ocorreram na direção da redução de quantidade de gordura corporal, em oposição à adoção de dietas hiperproteicas e hipercalóricas, assim como para o uso de EAA com a finalidade de aumentar a massa muscular.

Observou-se, no presente estudo, que o BSQ parece ser mais sensível às preocupações relacionadas à percepção corporal feminina, quando comparadas as expectativas perceptivas manifestadas pela amostra masculina. No entanto, - BSQ tem sido utilizado em avaliações da IC entre estudantes universitários de ambos os sexos (DI PIETRO, 200I) e adolescentes (CONTI; CORDAS; LATORRE, 2009). Ademais, em estudo que buscou identificar a validade das escalas brasileiras de avaliação da IC, verificou-se que os intervalos de pontuação do BSQ, usados para indicar níveis de preocupação com a IC, foram calculados em relação à média e desvio-padrão dos resultados da validação americana (CAMPANA; CAMPANA; TAVARES, 2009). Diante disso, sugere-se que futuros estudos sobre avaliação da IC sejam conduzidos focalizando estas limitações metodológicas.

A maioria dos casos de distúrbios de imagem corporal ocorre em mulheres jovens (HAY, 2002; OLIVA; FAGUNDES, 200I). No presente estudo, foi constatado que as professoras atuantes no fitness estão mais próximas de apresentarem distorção de IC (BSQ 7I,2 \pm 22,4) quando comparadas à amostra masculina (BSQ $53,2 \pm$ 17,0). Tal resultado pode ter sido influenciado pelo fato de o BSQ permitir avaliar a insatisfação pelo excesso de peso corporal, e não pelo baixo peso. No entanto, pesquisas confirmam a validade e a confiabilidade da versão brasileira do BSQ em universitários (DI PIETRO, 200 I ) e entre adolescentes de ambos os sexos (CONTI; CORDAS; LATORRE, 2009). 
Embora o caráter transversal do presente estudo não permita estabelecer relações de causa e efeito entre as variáveis independentes, como a (in) satisfação da imagem corporal dos investigados, foi possível observar que a autoexigência com o tamanho e a forma corporal, associado ao desejo de aceitação social e da eficácia técnica, parece estar contribuindo para intensificar os modos de inspeção da aparência corporal entre as professoras. Este achado vai ao encontro do estudo de Secchi, Camargo e Bertoldo (2009), em que as representações sociais do corpo e da IC de universitárias de diferentes cursos indicaram uma supervalorização da aparência nas relações pessoais e profissionais, revelando, ainda, que as estudantes analisadas associaram beleza e saúde corporal à magreza e à prática de exercícios físicos.

Em que pese a baixa prevalência de distorções de IC entre os (as) professores (as), observou-se uma constante preocupação e cuidado em torno da construção de uma forma ideal de corpo, suportando a idéia de que níveis elevados de desempenho físico e estético significam, sobretudo, garantia de domínio técnico, psicológico e mesmo moral aos olhos dos alunos (PALMA; ASSIS, 2005). Nessa direção, Espírito Santo e Mourão (2006) revelam que as imagens do professor e da professora de educação física aparecem no imaginário social como a de indivíduos imunes aos problemas de saúde. Seus corpos representam o ideal de saúde e as academias de ginástica são os principais espaços profissionais dessa representação. Assim, a inserção e a manutenção do professor e da professora no mercado de trabalho encontram-se influenciadas pelas exigências desse padrão. Diante do exposto, é possível considerar que tal sujeição por parte dos professores possa converter-se em sofrimento físico e psíquico, influenciando sua saúde e a socialização (NEUMARK-SZTAINER et al., 2006) além de sua empregabilidade.

$\mathrm{Na}$ avaliação dos escores do BSQ × IMC, os dados apontaram associação significativa somente para a amostra masculina, no entanto, esse resultado pode ter sido influenciado por não ter sido avaliado o nível de massa muscular. O IMC tem sido utilizado em estudos epidemiológicos para indicar sobrepeso (DAMASCENO et al., 2005; ALMEIDA et al., 2005), especialmente aqueles que apresentam grande tamanho amostral. O presente estudo, corroborando achados anteriores (COQUEIRO et al., 2008; FERMINO; PEZZINI; REIS, 20I0), indica que esta medida é frágil para avaliar (in) satisfação da imagem corporal e/ou de partes específicas dos corpos masculinos e femininos. Entretanto, existe evidência suportando a utilização do IMC na identificação da insatisfação com a IC (MILLSTEIN et al., 2008).

A aplicação de métodos epidemiológicos analíticos busca uma melhor compreensão sobre fatores ambientais, genéticos, sociais e econômicos associados ao risco do desenvolvimento de distúrbios psiquiátricos, uma vez que a avaliação da morbidade mostrou que este tipo de transtorno será considerado um dos principais 
problemas de saúde pública do século 2 I (HAY, 2002). Nessa direção, verificou-se que comportamentos associados à dieta hipocalórica têm maior chance de desenvolver distúrbios de IC entre os professores e as professoras. Tal resultado evidencia uma constante preocupação dos professores de educação física atuantes no fitness, em relação "à redução da quantidade de gordura corporal, visto que a gordura borra os contornos que delimitam os limites entre o corpo perfeito e um corpo fora dos padrões tanto estéticos como morais" (PALMA; ASSIS, 2005, p. 84).

Este resultado confirma o estudo de Thompson et al. (1995), ao associar o baixo peso corporal ao ideal de beleza feminina, assim como as expectativas masculinas de conquistar músculos fortes com baixo percentual de gordura. Grammas e Schwartz (2009) também apontaram que aspectos específicos de perfeccionismo parecem prever insatisfação com a IC em universitários do sexo masculino de diferentes nacionalidades. $O$ que corrobora com o achado de Seifert, Arnell e Kiviniemi (2008), ao revelar que as mulheres extremamente insatisfeitas com a IC foram as menos obesas.

Apesar do resultado referente à adoção da dieta hipercalórica, hiperproteica e também o uso dos EAA não afetar o desenvolvimento de distúrbios de IC, entre os professores do sexo masculino, atuantes e praticantes de musculação, o uso bioquímico parece ser um recurso precioso para alcançar rapidamente seus objetivos estéticos e/ou corporais (PALMA; ASSIS, 2005). Em uma visão antropológica, os autores ainda revelam que os efeitos virilizantes desses produtos parecem potencializar o aspecto competitivo no plano de trabalho e da vida cotidiana dos usuários. Tais recursos, adotados pelos professores do sexo masculino, corroboram com a pesquisa realizada por Carreira Filho e Martins Filho (2005), ao apontar maior interesse de modelagem corporal para o aumento de massa muscular entre os homens, quando comparado às mulheres. Do mesmo modo, no estudo de Halliwell, Dittmar e Orsborn (2007), praticantes de exercícios físicos do sexo masculino se sentiram motivados a aumentar a força e a massa muscular após exposição de imagens de homens musculosos. No entanto, Dolce et al. (1987) já apontavam que a percepção do tamanho do corpo pode não ser uma simples consequência da habilidade viso espacial, e sim de fatores cognitivos e afetivos em interação com habilidades perceptivas.

Observa-se que a variedade de condutas adotadas com o propósito de modelar o corpo reflete uma recorrente vulnerabilidade pessoal e ambiental que, ora menos, ora mais, parece contaminar tanto os frequentadores (HAY, 2002; GOELLNER, 2008), quanto os professores das academias de ginástica. Contudo, ao referir-se à regulação das relações humanas hipermodernas, o filósofo e sociólogo francês Gilles Lipovetsky (2005) aponta que a uniformização destes comportamentos, 
assim como a circulação dos corpos moldados, parece ir além do mero exibicionismo da perfeição física. Estes investimentos no corpo ampliam a rede de poderes que educam para o consumo de produtos e para a construção de representações de saúde, beleza e sucesso (GOELLNER, 2008). Para Lipovestsky (2005), o culto que se destina a corrigir partes específicas do corpo, tem também propósitos de maior adequação à personalidade, confiança em si mesmo e bem estar interior. No entanto, o desejo obsessivo por um ideal de musculosidade tem sido considerado uma das causas do abuso de EAA entre homens, ainda que tal prática provoque danos à saúde (CECCHETO, 2009).

Concluiu-se que a representação e os valores associados ao capital corporal do professor (a) de educação física atuante no ambiente do fitness parece ter significado expandido, apresentando-se como símbolos de saúde, competência, virilidade (PALMA; ASSIS, 2005), autocontrole, entre outros novos atributos, aparentemente necessários, que vêm configurar a imagem do professor.

\section{CONSIDERAÇÕES FINAIS}

Até onde foi possível verificar, os resultados sublinham as diferenças perceptivas em relação à insatisfação com a IC entre os professores e as professoras que atuam no segmento fitness. Apesar dos professores do sexo masculino não desconhecerem os danos do consumo das substâncias ergogênicas, eles se arriscam em prol de uma aparência idealizada. Ressalta-se que o desenvolvimento desta cultura masculina, associada à musculosidade exacerbada, assim como o desejo de conquistar o corpo cada vez mais magro, mesmo entre os profissionais de educação física atuantes em academias de ginásticas, deve ser considerado na concepção e gestão de programas de promoção da saúde.

Alem disso, a ideia de que as pessoas irão servir-se de seus corpos por intermédio de técnicas adquiridas, e que estas serão transmitidas de acordo com o meio no qual estão inseridas, possibilita a aquisição de um olhar crítico sobre o modo como os professores de educação física atuantes no fitness veem seus corpos, e a relação destes com as práticas corporais adotadas. De acordo com essa perspectiva, talvez seja possível perceber e ressignificar dar outros significados aos conceitos de saúde e beleza corporal que vigoram na atual sociedade ocidental. Por fim, sugere-se que novos estudos sobre a insatisfação da IC devam ser conduzidos, tendo por base fatores de natureza sociocultural que focalizem os ideais, as expectativas e experiências dos indivíduos investigados. 


\section{Evaluation of body image in physical education teachers working in the fitness market of the city of Rio de Janeiro}

This study aimed to identify the prevalence of body image distortions (IC) between genders in teachers working in the fitness market of the city of Rio de Janeiro. The Body Shape Questionnaire (BSQ) was used to evaluate the IC in 255 teachers ( 179 men). We found that $7.3 \%$ of male teachers and $32.8 \%$ of female teachers presented a slight or moderate distortion. Women (7I.2 \pm 22.4$)$ achieved statistically superior BSQ scores ( $p<0.001$ ) compared to men (53.2 \pm 17.0). There was also a growing concern and care for the construction of an ideal body shape among teachers, since high levels of physical and aesthetic performance ensure technical and psychological domain with the students, influencing their morale and success.

KEY WORDS: Diet; chemicals; teacher; aesthetics.

\section{Evaluación de la imagen corporal en profesores de educación física actuantes en el fitness de la ciudad de Rio de Janeiro}

RESUMEN: Identificar la prevalencia de distorciones de imagen corporal (IC) entre los sexos en profesores actuantes en el mercado de Fitness de la ciudad de Rio de Janeiro. Para la evaluación de la IC fue utilizado el Body Shape Questionnaire (BSQ) en 255 profesores (I 79 hombres). Observamos que 7,3\% de los profesores y 32,8\% de las profesoras mostraron una leve o moderada distorción. Las mujeres $(71,2 \pm 22,4)$ tuvieron resultados de BSQ estatisticamente superiores $(p<0,001)$ a los hombres $(53,2 \pm 17,0)$. Además se observó una creciente preocupación y cuidado con la construcción de una forma ideal del cuerpo entre docentes, porque niveles mas altos de rendimiento físico y estéticos significan una garantia de dominio técnico y psicológico con los alumnos, impactando en su moral y éxito.

PALABRAS CLAVES: Dieta; compuestos químicos; docentes; estética.

\section{REFERÊNCIAS}

ALMEIDA, G. A. N. et al. Percepção de tamanho e forma corporal de mulheres: estudo exploratório. Psicologia em Estudo, Maringá, v. 10, n. I, p. 27-35, jan./abr. 2005.

ALVARENGA, M. S. et al. Insatisfação com a imagem corporal em universitárias brasileiras. Jornal Brasileiro de Psiquiatria, Rio de Janeiro, v. 59, n. I, p. 44-5I, jan. 2010. Disponível em: <http://www.scielo.br/scielo.php?script=sci_arttext\&pid=S0047 $20852010000100007 \& \mid n g=e n \& n r m=i s o \& t \operatorname{lng}=p t>$. Acesso em: 10 set. 2009. 
BOSI, M. L. M. et al. Comportamento alimentar e imagem corporal entre estudantes de educação física. Jornal Brasileiro de Psiquiatria, Rio de Janeiro, v. 57, n. I, p. 28-33, jan. 2008. Disponível em: <http://www.scielo.br/pdf/jbpsiq/v57nl/v57n l a06.pdf> . Acesso em: 4 jan. 2009.

CAMPANA, A. N. N. B; CAMPANA, M. B; TAVARES, M. da C. G. C. F. Escalas para avaliação de imagem corporal nos transtornos alimentares. Avaliação Psicológica, Porto Alegre, v. 8, n. 3, p. 437-446, dez. 2009.

CARREIRA FILHO, D; MARTINS FILHO, J. Prevalência do uso de substâncias químicas entre adolescentes, com finalidade de modelagem corporal. Revista Brasileira de Ciências do Esporte, Campinas, v. 27, n. I, p. 93- I II, set. 2005.

CECCHETO, F. A sina de ser forte, sexualidade e saúde entre lutadores de jiu-jitsu no Rio de Janeiro. In: AQUINO, E. M. (Org.). Sexualidade, reprodução e saúde. Rio de Janeiro: Fundação Getúlio Vargas, 2009. p. 509-534.

CONTI, M. P; CORDAS, T. A; LATORRE, M. R. D. de O. Estudo de validade e confiabilidade da versão brasileira do Body Shape Questionnaire (BSQ) para adolescentes. Revista Brasileira de Saúde Materno Infantil, Recife, v. 9, n. 3, p. 33।-338, jul./set. 2009. Disponível em: <http://www.scielo.br/scielo.php?pid=SI 519-382920090003000 I 2\&script=sci abstract\&tlng $=$ pt $>$. Acesso em: 2 fev. 2010.

COQUEIRO, R. S. et al. Insatisfação com a imagem corporal: Avaliação comparativa da associação com estado nutricional em universitários. Revista de Psiquiatria do Rio Grande do Sul, Porto Alegre, v. 30, n. I, p. 31-38, jan./abr. 2008.

CORDÁS, T. A.; NEVES, J. E. P. Escalas de avaliação de transtornos alimentares. Revista de Psiquiatria Clinica, São Paulo, v. 26, n. I, p. 4I-47, jan./fev. 1999.

DAMASCENO, V. O. et al. Tipo físico ideal e satisfação com a imagem corporal de praticantes de caminhada. Revista Brasileira de Medicina do Esporte, Niterói, v. II, n. 3, p. |8|-186, maio/jun. 2005.

DI PIETRO, M. C. Validade interna, dimensionalidade e desempenho da escala BSQ - "Body Shape Questionnaire" em uma população de estudantes universitários. 2001 . 39 f. Dissertação (Mestrado em Ciências) - Escola Paulista de Medicina, Universidade Federal de São Paulo, São Paulo, 200l.

DOLCE, J. J. et al. Generalization of body size distortion. International Journal of Eating Disorders, Malden, v. 6, n. 3, p. 40I-408, may 1987.

ESPIRITO SANTO, G.; MOURÃO, L. A auto-representação da saúde dos (as) professores (as) de Educação Física de academias. Revista Brasileira de Ciências do Esporte, Campinas, v. 27, n. 3, p. 39-55, maio 2006. 
FERMINO, R. C.; PEZZINI, M. R.; REIS, R. S. Motivos para a prática de atividade física e imagem corporal entre frequentadores de academias. Revista Brasileira de Medicina do Esporte, Niterói, v. 16, n. I, p. 18-23, jan./fev. 2010.

GOELLNER, S. V. A construção dos corpos. Perspectivas Feministas. In: STEVEN, C. M. T.; SWAIN, N. (Org.). A cultura fitness e a estética do comedimento: as mulheres, seus corpos e aparências. Florianópolis: Mulheres, 2008. p. 245-280.

GOLDENBERG, M.; RAMOS, M. S. A Civilização das Formas: o corpo como valor. In:

(Org.). Nu \& vestido: dez antropólogos revelam a cultura do corpo carioca. Rio de Janeiro: Record, 2002, p. 19-40.

GRAMMAS, D. L.; SCHWARTZ, J. P. Internalization of messages from society and perfectionism as predictors of male body image. Body Image, Amsterdam, v. 6, n. I , p. 31 -36, jan. 2009.

HALLIWELL, E.; DITTMAR, H.; ORSBORN, A. The effects of exposure to muscular male models among men: exploring the moderating role of gym use and exercise motivation. Body Image, Amsterdam, v. 4, n. 3, p. 278-287, sept. 2007.

HAUSENBLAS, H. A.; FALLON, E. A. Exercise and body image: a meta-analysis. Psychology \& Health, Chur, v. 21, n. I, p. 33-47, feb. 2006.

HAY, P. J. Epidemiologia dos transtornos alimentares: estado atual e desenvolvimentos futuros. Revista Brasileira de Psiquiatria, São Paulo, v. 24, n. 3, p. 13-17, dez. 2002.

LEAL, V. C. L. V. et al. O corpo, a cirurgia estética e a saúde coletiva: um estudo de caso. Ciência \& Saúde Coletiva, Rio de Janeiro, v. 15, n. I, p. 77-86, jan. 2010.

LIPOVETSKY, G. A era do vazio: ensaios sobre o individualismo contemporâneo. Barueri: Manole, 2005.

LIZ, C. M. de. et al. Aderência a pratica de exercícios físicos em academias de ginástica. Motriz, Rio Claro, v. 16, n. I, p. 181-188, jan./mar. 2010.

MARANHÃO NETO, G. A. M; POLITO, M. D.; LIRA, V. A. Fidedignidade entre peso e estatura reportados e medidos e a influência do histórico de atividade física em indivíduos que procuram a prática supervisionada de exercícios. Revista Brasileira de Medicina do Esporte, São Paulo, v. II, n. 2, p. |4|-|45, mar./abr. 2005.

MILLSTEIN, R. A. et al. Relationships between body size satisfaction and weight control practices among US adults. Medscape Journal of Medicine, New York, v. 10, n. 5, p. I 19, may 2008.

MOREIRA, L. A. C. et al. Body image in a sample of undergraduate medical students from Salvador, Bahia. Jornal Brasileiro de Psiquiatria, Rio de Janeiro, v. 54, n. 4, p. 294-297, out./ dez. 2005. 
NEUMARK-SZTAINER, D. et al. Does body satisfaction matter? Five year longitudinal associations between body satisfaction and health behaviors in adolescent females and males. Journal of Adolescent Health, San Francisco, v. 39, n. 2, p. 244-25 I, aug. 2006.

OLIVA, C. A. G.; FAGUNDES, U. Aspectos clínicos e nutricionais dos transtornos alimentares. Psiquiatria na Prática Médica, São Paulo, v. 34, n. 2, p. 47-53, abr./jun. 2001.

OLIVEIRA, F. P. et al. Comportamento alimentar e imagem corporal em atletas. Revista Brasileira de Medicina do Esporte, São Paulo, v. 9, n. 6, p. 348-369, nov./dez. 2003.

PALMA, A.; ASSIS, M. Uso de esteróides anabólico-androgênicos e aceleradores metabólicos entre professores de educação física que atuam em academias de ginástica Revista Brasileira de Ciências do Esporte, Campinas, v. 27, n. I, p. 75-92, set. 2005.

SARDINHA, A.; OLIVEIRA, A. J.; ARAÚJO, C. G. S. Dismorfia muscular: análise comparativa entre um critério diagnóstico e um instrumento psicológico. Revista Brasileira de Ciências do Esporte, Campinas, v. I4, n. 4, p. 387-392, jul./ago. 2008.

SECCHI, K; CAMARGO, B. V.; BERTOLDO, R. B. Percepção da imagem corporal e representações sociais do corpo. Psicologia: teoria e pesquisa, Brasília, v. 25, n. 2, p. 229-236, abr.jun. 2009.

SEIFERT, A. L.; ARNELL, K. M; KIVINIEMI, M. T. The relation of body dissatisfaction to salience of particular body sizes. Eating Weight Disorder, Lincoln, v. 13, n. 4, p. 84-90, dec. 2008.

SILVA LOPES, V. M. B.; CHIAPETA, S. M. S. V. Motivos de adesão e manutenção da prática de atividades físicas regulares em academias de ginástica de Ubá/MG. Revista Digital, Buenos Aires, v. 15, n. 143, abr. 20 10. Disponível em: <http://,efdeportes.com>. Acesso em: 24 set. 2011 .

THOMPSON, J. K. et al. The Perception of Teasing Scale (POTS): revision and extension of the Physical Appearance Related Teasing Scale (PARTS). Journal of Personality Assessment, Hillsdale, v. 65, n. I, p. |46- I57, aug. 1995.

Recebido: 26 abr. 20II

Aprovado: 23 out. 2011

Endereço para correspondência:

LígiaVilhena

Avenida Brasil, 4365, Pavilhão Lauro Travassos, sala 28, Manguinhos, Rio de Janeiro, RJ, Brasil CEP: 21040.900 